\title{
8th Asian Biological Inorganic Chemistry Conference
}

\author{
Christian G. Hartinger ${ }^{1}$
}

Published online: 2 June 2017

(C) SBIC 2017

As the regional analogue of the ICBIC series, AsBIC conferences address the important issues at the forefront of Biological Inorganic Chemistry, with a special emphasis on the developments coming out of the Asia-Pacific region. The previous AsBIC conferences were held in Okazaki (2003/03), Goa (2004/12), Nanjing (2006/10), Jeju (2008/11), Kaohsiung (2010/11), Hong Kong (2012/11), and Gold Coast (2014/11). The 8th Asian Biological Inorganic Chemistry Conference (AsBIC-8), chaired by Professor Christian G. Hartinger, was held at the University of Auckland, New Zealand, December 4-9, 2016, where we welcomed around 340 bioinorganic chemists from 25 countries. The topics covered at the conference included Metals in Medicine, Metalloproteins and Energy, Bioinspired Chemistry, and Metallomics and Nanotechnology in Bioinorganic Chemistry, to name a few. The conference featured 7 plenary, 3 award, 25 keynote, and 110 invited lectures, as well as 54 oral and 110 poster presentations. The inaugural Graeme Hanson Early Career Researcher Awards were presented to Dr. Anna Renfrew (University of Sydney) and Dr. Ho Yu Au-Yeung (University of Hong Kong), while Prof. Shunichi Fukuzumi received the AsBIC Outstanding Achievement Award 2016. The contributors to this special issue gave fascinating plenary, award, or keynote lectures at the conference, and the breadth of research conducted and presented at the AsBIC conferences is also reflected in this special issue.

Christian G. Hartinger

c.hartinger@auckland.ac.nz

1 School of Chemical Sciences, The University of Auckland, Private Bag 92019, Auckland 1142, New Zealand
A significant number of contributions focused on imaging and sensing techniques in Bioinorganic Chemistry. In this issue, Kazuya Kikuchi summarizes recent developments of fluorescent dyes for super-resolution microscopy (doi:10.1007/s00775-016-1435-y), while Fuyi Wang uses secondary ion mass spectrometry imaging to visualize metallodrugs in single cells (doi:10.1007/s00775-0171462-3). Peter Lay reports studies on the reason for the high cytotoxicity observed for vanadium(IV) complexes (doi:10.1007/s00775-017-1453-4), while Hongzhe Sun and Hongyan Li investigate the mechanism of action responsible for the antimicrobial activity of bismuth(III) compounds (doi:10.1007/s00775-017-1452-5). Peng Chen reports on his group's efforts to obtain crystal structures of DNA-bound forms of multiple-antibiotic-resistance regulator (MarR), which controls, among other things, bacterial resistance to antibiotics (doi:10.1007/s00775-017-1442-7).

As in many Biological Inorganic Chemistry conferences, significant contributions to the understanding of metalloproteins and their biological functions were reported. This special issue includes Takashi Hayashi's work on the reconstitution of horseradish peroxidase with cobalt tetradehydrocorrin to obtain a cobalamin-dependent enzyme model (doi:10.1007/s00775-017-1458-z). Shun Hirota discusses the effect of Met80 heme coordination on cyt $c$ domain swapping (doi:10.1007/s00775-017-1446-3), and Mio Kondo and Shigeyuki Masaoka describe iron porphyrin complexes as bioinspired catalysts for $\mathrm{CO}_{2}$ reduction (doi:10.1007/ s00775-017-1438-3), while Jing Zhang and Jun-Long Zhang present studies on linear tetrapyrrole as ligands and analogues to porphyrin and corroles (doi:10.1007/s00775-0171444-5). The reactivity of other important biological ligands, i.e., catechols, in siderophores and synthetic analogues as well as of Dopa-containing mussel foot proteins is reviewed by Alison Butler (doi:10.1007/s00775-017-1451-6). 


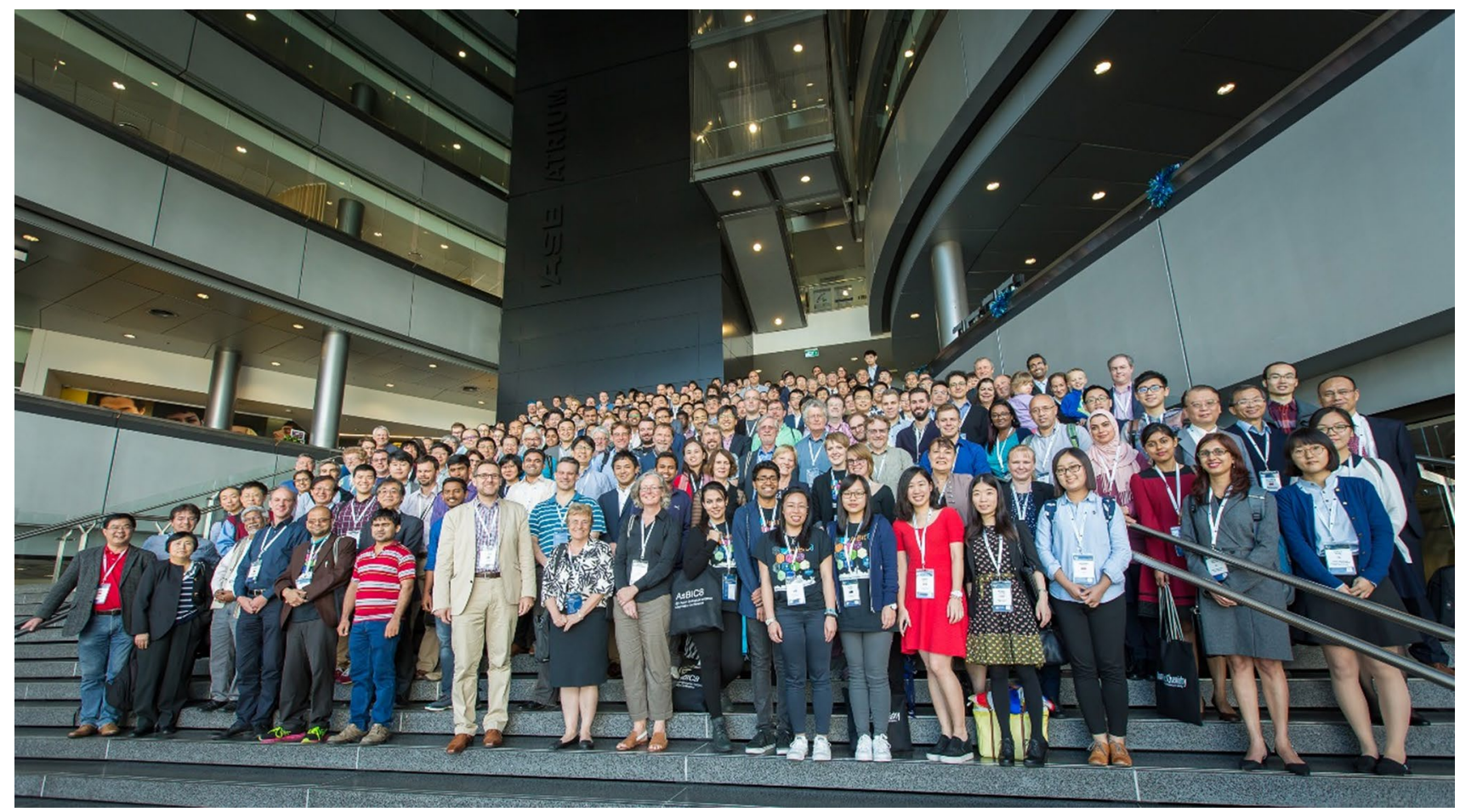

In a contribution on Se speciation in the environment, Debbie Crans points out the importance of speciation for toxicity (doi:10.1007/s00775-017-1457-0). Elizabeth New and Trevor Hambley present their work on the cisplatin interaction with the copper transporter CTR1, which has been shown to play a role in the uptake of cisplatin into cancer cells (doi:10.1007/s00775-017-1467-y), and finally, both Paul Bernhardt's group and my group report work on Co complexes as tools for the spectroelectrochemical investigation of heme proteins and for capillary zone electrophoresis, respectively (doi:10.1007/s00775-016-1427-y, doi:10.1007/s00775-016-1426-z).

I hope to see many of you again at AsBIC-9 in Singapore in 2018 (http://www.asbic9.org) and that you enjoy the selection of papers presented in this special issue of the Journal of Biological Inorganic Chemistry. 